\title{
Suicide prevention: a proposed national strategy for South Africa
}

\author{
L Schlebusch \\ Emeritus Professor of Behavioural Medicine, University of KwaZulu-Natal, Durban, South Africa
}

\begin{abstract}
Suicidal behaviour is an important public health problem globally and in Africa. A brief overview of the nature and severity of the problem is provided, but the primary aim of this paper is to identify priorities and prevention strategies for reducing suicidal behaviour in South Africa by discussing a framework for a proposed national prevention programme. South African suicide rates range from 11.5 per 100000 to as high as 25 per 100000 of the population, depending on sampling procedures and research methods. About 1 1\% of all non-natural deaths are suicide related. On average $9.5 \%$ of non-natural deaths in young people are due to suicide. It is a complex phenomenon and risk factors are, therefore, multifactorial and multidimensional. Some of the most important ones are identified and several priorities and prevention possibilities for reducing suicidal behaviour are recommended. The outline and structure for such a national suicide prevention programme is underpinned by research undertaken locally and internationally. It requires a comprehensive multi-sectoral approach that involves both health care and non-health care sectors and action at various levels utilising a framework based on a set of guiding principles and a range of strategies with specific objectives as a national priority within an interdisciplinary context.
\end{abstract}

Keywords: Suicide; Prevention; National strategy; South Africa

Received: $16 / 08 / 2012$

Accepted: 14/09/2012

doi: http://dx.doi.org/10.4314/ajpsy.v15i6.56

This article is based on a paper presented at the National Mental Health Summit convened by the Department of Health, Republic of South Africa from 12-13 April 2012.

\section{Introduction}

Suicidal behaviour is an important public health problem globally ${ }^{1}$ and in Africa. ${ }^{2}$ The available statistics are only the tip of the iceberg, and given their devastating consequences, prevention efforts are essential. The primary aim of this paper is to identify priorities and prevention strategies for reducing suicidal behaviour in South Africa by discussing a framework for a proposed national prevention programme. To accomplish this, it is necessary to provide a brief overview of the nature and severity of the problem.

\section{Epidemiology}

Globally, suicide rates have increased by $60 \%$ in the last few decades, and the World Health Organization (WHO) estimates a worldwide yearly suicide mortality rate of almost one

\section{Correspondence}

Professor L Schlebusch

Post Net Suite 208, Private Bag x10, Musgrave Road, 4062

Durban, South Africa

email: schlebusch@ukzn.ac.za million people, which is projected to increase to 1.5 million by 2020. ${ }^{1}$ Between 10 and 40 times more people engage in nonfatal suicidal behaviours (attempts). The predominance of male suicide rates over females has been relatively constant, i.e. an approximate ratio $=3.6: 1$. An exception to this is in rural China, where female rates are higher than male rates. Suicides in the world amount to $1.4 \%$ of the total mortality and $15 \%$ of the injury mortality figures. The global suicide rate is estimated at 11.6 to 16 per 100,000 inhabitants (18 per 100.000 for males and 11 per 100.000 for females). Suicide mortality has moved from Western Europe to Eastern Europe and now seems to be shifting to Asia, while China and India are the biggest contributors to the absolute number of suicides in the world. ${ }^{1,3-4}$

African suicide statistics are equally alarming, but due to a lack of reliable data, the full extent of its burden has not been well understood in the past.2,5-6 In South Africa, three important research-based efforts have provided a broader picture of suicidal behaviour. The first is a multicentre programme targeting non-fatal suicidal behaviours, known as the Durban Parasuicide Study (DPS) under the leadership of the author (L S) which has generated several conferences and considerable data. 5,7 The second focuses on fatal suicidal behaviour and commenced with the development in 1999 of the National Injury Mortality Surveillance System (NIMSS). ${ }^{8}$ Information for this system is collated from existing 
investigative procedures at mortuaries and state forensic laboratories, as part of a collaborative effort between different research groups and government bodies in South Africa. A third is the South Africa Stress and Health study $(\mathrm{SASH})^{9}$ based on a large epidemiological survey of nationally representative data. Data from the DPS, the NIMSS, SASH and other individual studies ${ }^{2,5,7-8}$ provide a disturbing profile of suicidal behaviour in South Africa, noting that it is inordinately high in all age groups. Although figures reflect only a part of the problem with diverse reported prevalence rates, there is a genuine escalation of the problem, rather than simply a reflection of improved recording practices over recent years. Nevertheless, data must be interpreted with caution when making cross-national, cross-cultural and cross regional comparisons. . $^{2,5,7}$

South African suicide rates ${ }^{2,5,7}$ range from 11.5 per 100000 to as high as 25 per 100000 of the population, depending on sampling procedures and research methods. The estimated fatal to non-fatal ratio $=1: 20$. In some occupational groups such as in the South African Police Service, the figures are higher. About $11 \%$ of all non-natural deaths are suicide related. On average $9.5 \%$ of non-natural deaths in young people are due to suicide, which is almost as high as the adult suicide rates. These rates translate into approximately one to two suicides and 20 or more attempts per hour. Suicides occur predominantly in males with an approximate male to female ratio $=5: 1$. In non-fatal suicidal behaviours females predominate with an approximate female to male ratio $=3: 1$. Regarding fatal suicidal behaviour the average age is 34 years, which is consistent with global findings of a shift from the elderly to younger people. Suicides in people as young as $<10$ years old have been reported, although most suicides in the young are in 15-19 year age group. In non-fatal suicidal behaviour, the peak age is in the 20-29 age group with a mean age of 25 years. About one third of hospital admissions for non-fatal suicidal behaviour involve children and adolescents. Generally the child and adolescent group is the second most at-risk age group for non-fatal suicides after young adults. Non-hospital based studies report suicidal behaviour / ideation in school children that range between $4 \%$ and $47 \%$ . The most important methods used in suicide are: hanging (most frequent), shooting, poisoning / overdosing, gassing, and burning. Other methods include using: sharp objects, asphyxia, electrocutions, drowning,

falls/fenestrations(jumping off high areas), and hypoxiphilia / auto-erotic asphyxiation. The main choice of methods in non-fatal suicidal behaviour includes about $90 \%$ overdose and 10\% self-injury. Overdose generally involves ingesting household poisons/cleaning agents, over-the-counter analgesics, benzodiazepines, and anti-depressants. Suicidal behaviour occurs mostly over weekends between 7am and 8pm, although there are exceptions to this. Some seasonal variations over the year have been reported, with peaks around year end (often because of exam stress and attempts to enter university or the open labour market). 2,5,7-8

\section{Risk factors}

Suicidal behaviour is a process, with suicidal ideation forming part of its evolution. It is a complex phenomenon and risk factors are, therefore, multifactorial and multidimensional. Some of the most important ones identified in South African studies, $2,5,7$ are briefly mentioned here, as space does not allow for an in-depth discussion of the various pathways to suicidal behaviour.

\section{Imitation effects and suicidal transmission}

The influence of the mass media and information technology on vulnerable people can precipitate suicidal behaviour because of imitation or copycat effects. Websites exist that graphically describe suicide methods. The internet, information and communication technology development have created expanded opportunities to influence vulnerable people thereby enhancing the contagious effects of suicidal behaviour (the Werther effect).

\section{Dysfunctional problem-solving skills}

Suicidal people are often poor at problem solving skills and dealing with interpersonal problems. In this context suicidal behaviour has been viewed as an inappropriate problemsolving strategy and method of communication, especially by vulnerable people when they feel unable to express their distress in a conventional manner or if other attempts to deal with their problems have been unsuccessful. ${ }^{5}$ Further, at least $80 \%$ of suicidal behaviours are preceded by either verbal or non-verbal behavioural cues that indicate the suicidal person's intentions. The commonly held belief, therefore, that people who threaten to commit suicide are not serious about it is an artifact. Repeated suicidal behaviour can increase in order to secure help when suicide attempts do not get the desired effect from significant others on whom the suicidal behaviour is supposed to impact (i.e. if the cry for help fails).

\section{Neuropsychological correlates}

Head injury and/or neuropsychological/cognitive deficits can be related to aggressiveness/impulsivity/poor decision making and brain pathology can trigger depression, disinhibition or lack of restraint and subsequent suicidal ideation or behaviour. Additionally, significant correlates of suicidal behaviour have been associated with personality functioning involving substance abuse, emotional lability, aggression and impulsivity.

\section{Neurobiological correlates}

Neuroimaging is enhancing the understanding of biological vulnerability in suicidal behaviour. Reduced serotonergic input to the orbital pre-frontal cortex (part of the brain that is involved in behavioural inhibition and decision making) may result in aggressiveness/impulsivity. If, there are decreases in pesynaptic binding sites in the prefrontal cortex (altered receptor population), serotonergic hypofunction may also be associated with more lethal methods of choice in suicidal behaviour.

\section{Violence}

Themes that overlap in this respect include: increased aggressiveness, impulsiveness, emotional lability, disinhibition, dysfunctional decision-making and reasoning In addition, an underlying biological or genetic predisposition could result in increased aggressiveness and violent acting out resulting in suicidal behaviour. 


\section{Genetics and familial transmission}

Patients who engage in suicidal behaviour often have a higher rate of suicidal behaviour and related

psychopathology such as mood disorders and substance abuse in their families. Twin studies have demonstrated a higher concordance rate for suicidal behaviour in monozygotic compared to dizygotic twins.

\section{Stress}

Acute and chronic stress are critical co-morbid aetiological variables, and of particular important in South African society. Several stress models, with significant advantages for treatment and prevention have been advanced. Two important such models are a stress-diathesis mode ${ }^{10}$ and a stress-vulnerability model. ${ }^{11}$ Both are applicable to the South African situation

\section{Diet \\ Dietary inefficiency can affect suicidality because of potential effects on neurotransmitter functioning. Micronutrient deficiencies can have adverse psycho- physiological consequences by progressively reducing stress tolerance and impact adversely on brain structures. This in turn may impair appropriate coping strategies in a suicidal person.}

\section{Somatic potentially life-threatening diseases}

An elevated suicide risk in vulnerable patients with certain somatic diseases has been found. ${ }^{12}$ For example, one in four South Africans develop cancer and one in two is likely to know someone that has cancer. Suicide risk can occur in these patients because the disease can constitute a life crisis resulting in a gamut of psychological problems. ${ }^{13} \mathrm{Also}$, childhood cancer can have a long term suicide risk manifesting in adults due to traumatic hospitalisation, depression and altered social contact as children. ${ }^{13}$ In South Africa, HIV-AIDS sufferers have been shown to have a high suicide risk under certain conditions. ${ }^{5,14-15}$

\section{Other common precipitators}

These include: interpersonal problems, family/marital problems, partner-relational problems, extended suicides/family murders, financial/socio-economic problems, academic-related problems, incest, exposure to family violence, child abuse and most importantly psychiatric/psychological disorders (especially mood disorders and alcohol/drug abuse).

\section{Recommended prevention initiatives}

The outline and structure for a national suicide prevention programme recommended here is underpinned by research undertaken locally ${ }^{5,7}$ and internationally as based on existing initiatives for suicide prevention from several countries. ${ }^{16}$ In addition, several recommended priorities and prevention possibilities for reducing suicidal behaviour in Africa and South Africa ${ }^{7,17-18}$ form the basis of much of the following discussion. The high suicidal behaviour prevalence rates have considerable implications for mental health-care facilities in the country. It is important to monitor these patterns on an ongoing basis as evidence shows that suicidal behaviour in different groups changes across time, and there is a need to develop appropriate, cost-effective preventions. Some local and regionalised suicide prevention initiatives exist which include the following: (a) Government strategies that are intended to draw together various government departments and other efforts as discussed at the National Health Summit held on 12-13 April 2012. These should also aim at: (i) broadening the public's awareness of suicide and its risk factors, (ii) enhancing population-based and clinical care services/programmes, policy guidelines on child and youth mental health, and life skills programmes; and (iii) suicide prevention through effective monitoring systems and research. (b) The South African Depression and Anxiety Group (SADAG), which serves as an advocacy and support network for those suffering from Depression, Bipolar Mood Disorder, Panic Disorder, Post-Traumatic Stress Disorder, Social Anxiety Disorder, Obsessive Compulsive Disorder, and Generalised Anxiety Disorder. It is a non-profit organisation and has a board consisting of psychiatrists, psychologists and general practitioners. Free telephonic counselling is a major focus, with a referral service to experts and medical treatment where appropriate. Specific attention is directed also at media campaigns to de-stigmatise mental illness and to encourage people to come forward for treatment. SADAG has worked in many schools assisting in teen suicide prevention programmes. (c) Life Line Southern Africa (affiliated to LifeLine International) which provides a free 24 hour crisis intervention service available to all sectors of the community. The service is primarily based on a telephone counselling service. (d) The S A - Federation for Mental Health which co-ordinates, monitors and promotes services for persons with Intellectual Disability, psychiatric disability as well as mental health well being. (e) The International Association for Suicide Prevention (IASP) which is represented in South Africa and which forms an important link between local and international initiatives. (f) The Mental Health Information Centre of South Africa, based at the Department of Psychiatry at the University of Stellenbosch which forms part of the Medical Research Council's Unit on Anxiety and Stress Disorders. (g) The World Health Organization which has drafted strategy proposals for suicide preventive work and published a series of documents on how to prevent suicide in psychiatric and general practice settings, in schools, prisons and in survivors of suicide, and how to report suicide in the mediaall of which can be applied to the South African setting.

However, no national prevention programme exists. Such a programme for South Africa has been proposed before ${ }^{17}$ and requires a comprehensive multi-sectoral approach that involves both health care and non-health care sectors. Critical key prevention strategies of relevance in the South African setting recommended are: recognition of risk factors; awareness-raising; dissemination of information on suicidal behaviour; effective monitoring; improved data collection; collaborative research; more access to treatment of comorbid psychiatric/psychological conditions by well-trained health-care practitioners; the development and enhancement of community-based efforts; restricting access to the methods of suicide; wide dissemination and exchange of data and research; improved inter-African collaborative research; networking and global co-operation; access to the 
latest information about suicide prevention; and the fact that all strategies must be appropriate to the social and cultural needs of the diverse populations they serve. The following additional considerations are important.

\section{Primary health care}

Because psychiatric/psychological conditions and substance dependence/abuse have been significantly associated with increased suicidal behaviour risk, more attention should be given to their early detection and effective treatment within primary health care settings. Treatment strategies must be holistic and culturally relevant and consider traditional beliefs. The aim should be to create uniquely African strategies, not simply to transplant knowledge on suicide prevention into Africa from the Western world. ${ }^{5}$

\section{Further education and training}

Those who come into contact with suicidal individuals require further education and training programmes to help eliminate myths surrounding suicidal behaviour and to provide information about early recognition of relevant symptoms. Promoting skills development on how to support and assist suicidal patients and their families in obtaining help form a necessary part of such programmes, which should also target government departments, the police, the legal profession, the clergy and other relevant role players. Because of the high levels of suicidal behaviour among the youth in South Africa, school personnel and parents are of particular importance in this regard. ${ }^{7}$

\section{Means Restriction}

There is international evidence that controlling access to and the availability of methods used in suicide can be an effective strategy in suicide prevention. ${ }^{16,19}$ As noted, in South Africa, overdose is a common choice of method. This involves the ingestion of pesticides, common household utilities (paraffin, poisons, etc.), prescription and over-the-counter medications. In addition, hanging, gassing, burning, jumping from buildings or high places (such as bridges) and firearms are among the most frequently used methods in suicidal behaviour. There is also a direct link between suicide and alcohol and other substance abuse. Access to all these methods typically differ substantially across socio-demographic groups. Modifying policies to control their production, distribution and sales; promoting their safer storage; improving the medical management of pesticide/poison ingestion; gun control; and educating the community are recommended as they may have dramatic effects on reducing the risk of suicidal behaviour in the country. A number of factors influence the choice of method used such as: a) accessibility thereto; b) knowledge or lack of the lethality of the method; c) experience and familiarity with the method; d) the meaning, symbolism and cultural influence involved; e) the suicidal person's state of mind; f) the level of intent to succeed or not. ${ }^{5}$ All these factors need to be considered when designing prevention programmes.

\section{Imperative action at various levels}

Intervention at the individual/family level is obvious, given the role of family problems/psychopathology and interpersonal conflicts in suicidal behaviour. However, action at societal level is also essential. Child safety and the plight of vast members of South African children, traumatised citizens, high prevalence rates of violence and trauma, first world influences in an internationally less isolated post apartheid South Africa, high expectations which are not always realized, acculturation, socio economic difficulties including high unemployment levels and economic pressures ${ }^{5,7}$ which (if not timeously addressed) all combine to produce a potential breeding ground for suicidality. These variables need to be addressed at a societal level as part of a suicide prevention programme. Action at the community level is necessary, given the limited numbers of mental health professionals. Community-based initiatives such as mental health centres and education/training programmes can play an essential role in the prevention of suicidal behaviour. Suicide crisis centres provide critical immediate emotional support to and referral bases for suicidal people and their families. Following their bereavement after a suicide, help for families is essential, and post-vention recommendations are based on preferred early contact with the family. ${ }^{7}$ Self-help support groups are potent, inexpensive and constructive ways for people to help themselves and others. A community's perception of suicidal behaviour can play an important role in preventing suicide or the degree to which suicidal behaviour is disguised. The media can strongly influence these perceptions and there is evidence to suggest that over-dramatized media presentations of suicide can increase suicide rates. Encouraging responsible media reporting is essential in this regard. 5,16 Studies, regarding socio-economic influences on suicide have shown mixed results, but people living in low socio-economic conditions have been found to be at increased risk of suicide. ${ }^{7}$ In some research ${ }^{5}$, social fragmentation has been noted to be more strongly associated with suicide than deprivation per se, and in recent years, there has been an increased focus on the global economic meltdown and increased suicide following decreased social support and community connectedness. For example, following the recent economic crises in Europe a rise in unemployment was associated with an increase in suicides in people younger than 65 years $^{20}$ and in Greece there has been an annual increase of $40 \%$ in suicides. ${ }^{21}$

\section{Conclusion}

There should be recognition of the fact that a comprehensive approach to suicide prevention requires a framework based on a set of guiding principles and a range of strategies to achieve specific objectives. In addition to incorporating the considerations already elucidated thus far, a national programme for suicide prevention should endeavour to provide a strategic framework for action at all levels to prevent suicidal behaviour and promote mental health. Identification of risk factors is critical to prevent suicidal behaviour in all groups of the population within their diverse settings. Specific goals should aim to: reduce fatal and nonfatal suicidal behaviour by reducing risk factors; promote protective factors; reverse emerging problems areas; focus on early detection of new trends in suicidal behaviour; promote public awareness of suicidal behaviour, its causes and possibilities for prevention; and increase support available to individuals, families and communities affected 
by suicidal behaviour. Partnerships and alliances within the community need to be created and support provided for professional groups and NGOs. There should be a focus on: a diversity of approaches; building on existing strengths, capacities and capabilities; creating an evidence-based and outcome-focused programme, of which evaluation should form an integral part; developing activities that are appropriate and responsive to the social and cultural needs of the groups or populations they serve; developing a rights based approach; ongoing improvement of national suicidal behaviour data collection systems and monitoring trends; addressing social attitudes by mental health-promotion and increasing knowledge of suicidal behaviour to reduce stigma and discrimination. Targeting specific groups are important, such as, for example, children, young people, the elderly and victims of violence.

Finally, the burden of suicidal behaviour in South Africa underscores the need for suicide prevention as a national priority. Such a strategy should be implemented in a coordinated and strategic manner within an interdisciplinary context, and any suicide prevention efforts need to take cognisance of the numerous stresses that a developmental state in transition presents to its citizens.

\section{References}

1. Bertolote JM, Fleischmann A. A global perspective on the magnitude of suicide mortality. In: D. Wasserman, \& C Wasserman, eds, Oxford Textbook of Suicidology and Suicide Prevention. A Global Perspective. Oxford: Oxford University Press, 2009, 91-98.

2. Schlebusch L. An overview of suicidal behaviour in Africa. In: Ndetei DM, \& Szabo CP, eds, Contemporary Psychiatry in Africa: A Review of Theory, Practice and Research. Nairobi, Kenya: Acrodile Publishing, 2011, 375-396.

3. Bertolote JM, Fleischmann A, De Leo D, Wasserman D. Suicidal thoughts, suicide plans and attempts in the general population on different continents. In: D. Wasserman, \& C. Wasserman, eds, Oxford Textbook of Suicidology and Suicide Prevention. A Global Perspective. Oxford: Oxford University Press, 2009, 99-104.

4. Varnik P. Suicide in the world. Int. J. Environ. Res. Public Health 2012; 9(2): 521-530.

5. Schlebusch L. Suicidal Behaviour in South Africa. Pietermaritzburg: University of Kwa-Zulu Natal Press, 2005

6. Burrows S, Schlebusch L, Musisi S, Kinyanda E, Kizza R. Selfdirected violence. In: Kobusingye O, Bowman B, Burrows $S$, Matzopoulos R, Butchart A, eds, Violence and Health in the WHO African Region. Regional Office for Africa, Brazzaville: World
Health Organization, 2011, 119-161.

7. Schlebusch L. Suicidal behaviour. In: van Niekerk A, Suffla S, Seedat $M$, eds, Crime, Violence and Injury in South Africa: 21 st Century Solutions For Child Safety. Houghton, Johannesburg, Psychological Society of South Africa: 2012, 178-194.

8. NIMSS. A profile of fatal injuries in South Africa.7th Annual Report. 2007. http://www.doh.gov.za/docs/report/2007/nimss/secla.pdf

9. Joe S, Stein DJ, Seedat S, Herman A, Williams DR. Non-fatal suicidal behavior among South Africans: Results from the South Africa Stress and Health Study. Soc. Psychiatry Psychiat Epidemiol 2008; 43: 454461.

10. Mann JJ. A current perspective of suicide and attempted suicide. Ann Intern Med 2002; 136: 302-311.

11. Wasserman D. A stress vulnerability model and the development of the suicidal process. In: D. Wasserman, ed, Suicide: An Unnecessary Death, London: Martin Dunitz, 2001, 13-27.

12. Berman L, Pompili M, eds. Medical Conditions Associated with Suicide Risk. American Association of Suicidology: Washington, 2011.

13. Schlebusch L. Suicide risk and cancer. In: Berman L, Pompili M, eds, Medical Conditions Associated with Suicide Risk. Washington: American Association of Suicidology; 2011, 59-74.

14. Govender RD, Schlebusch L. Suicidal ideation in seropositive patients seen at a South African HIV Voluntary Counseling and Testing Clinic. Afr J Psychiat 2012;15: 94-98

15. Govender RD, Schlebusch L. Hopelessness, depression and suicidal ideation in HIV-positive persons. S Afr J Psychiat 2012; 18: 17-21.

16. Wasserman D, Wasserman C, eds. Oxford Textbook of Suicidology and Suicide Prevention. A Global Perspective. Oxford: Oxford University Press, 2009; 723-850.

17. Burrows S, Schlebusch L. Priorities and prevention possibilities for reducing suicidal behaviour. In: Van Niekerk A, Suffla S, Seedat, $M$ (Eds). Crime, Violence and Injury Prevention in South Africa: Data to Action. Tygerberg: MRC-UNISA, 2008, 173-193.

18. Burrows S, Schlebusch L. Suicide prevention in South Africa. In: D. Wasserman, \& C. Wasserman (Eds). Oxford Textbook of Suicidology and Suicide Prevention. A Global Perspective. Oxford: Oxford University Press, 2009, 755-757.

19. Yip PSF, Caine E, Yousuf S, Chang SS, Wu KCC, Chen YY. Means restriction for suicide prevention. Lancet 2012; 379 (9834): 23932399.

20. Stuckler D, Basu S, Surcke M, Coutts A, Mckee M. Effects of the 2008 recession on health: a first look at European data. Lancet 201 1; 378:124-125.

21. Economou M, Madianos M, Theleritis C, Peppou LE, Stefanis, CN. Increased suicidality amid economic crisis in Greece. Lancet 2011; 378:1459

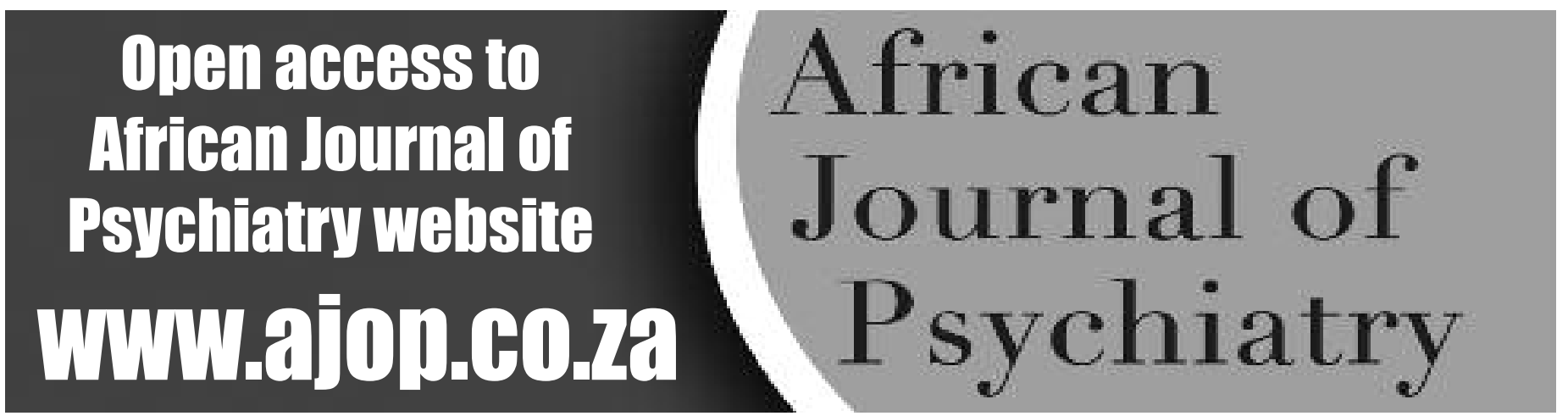

